\title{
Merotelic kinetochore orientation versus chromosome mono-orientation in the origin of lagging chromosomes in human primary cells
}

\author{
Daniela Cimini ${ }^{1,2}$, Daniela Fioravanti ${ }^{1}$, E. D. Salmon ${ }^{2}$ and Francesca Degrassi ${ }^{1, *}$ \\ ${ }^{1}$ Center for Evolutionary Genetics CNR, c/o Department of Genetics and Molecular Biology, University 'La Sapienza', Via degli Apuli 4, \\ 00185 Rome, Italy \\ 2Department of Biology, CB\#3280, University of North Carolina at Chapel Hill, Chapel Hill, NC 27599, USA \\ *Author for correspondence (e-mail: f.degrassi@caspur.it) \\ Accepted 1 November 2001 \\ Journal of Cell Science 115, 507-515 (2002) (C) The Company of Biologists Ltd
}

\section{Summary}

Defects in chromosome segregation play a critical role in producing genomic instability and aneuploidy, which are associated with congenital diseases and carcinogenesis. We recently provided evidence from immunofluorescence and electron microscopy studies that merotelic kinetochore orientation is a major mechanism for lagging chromosomes during mitosis in PtK1 cells. Here we investigate whether human primary fibroblasts exhibit similar errors in chromosome segregation and if at least part of lagging chromosomes may arise in cells entering anaphase in the presence of mono-oriented chromosomes. By using in situ hybridization with alphoid probes to chromosome 7 and 11 we showed that loss of a single sister is much more frequent than loss of both sisters from the same chromosome in anatelophases from human primary fibroblasts released from a nocodazole-induced mitotic arrest, as predicted from merotelic orientation of single kinetochores. Furthermore, the lagging of pairs of separated sisters was higher than expected from random chance indicating that merotelic orientation of one sister may promote merotelic orientation of the other. Kinetochores of lagging chromosomes in anaphase human cells were found to be devoid of the mitotic checkpoint phosphoepitopes recognized by the 3F3/2 antibody, suggesting that they attached kinetochore microtubules prior to anaphase onset. Live cell imaging of H2B histone-GFP-transfected cells showed that cells with mono-oriented chromosomes never enter anaphase and that lagging chromosomes appear during anaphase after chromosome alignment occurs during metaphase. Thus, our results demonstrate that the mitotic checkpoint efficiently prevents the possible aneuploid burden due to mono-oriented chromosomes and that merotelic kinetochore orientation is a major limitation for accurate chromosome segregation and a potentially important mechanism of aneuploidy in human cells.

Key words: Lagging chromosomes, Merotelic orientation, Monoorientation, Mitotic checkpoint, Aneuploidy

\section{Introduction}

Mitotic chromosome malsegregation produces aneuploidy and genome instability. An increasing number of studies have shown that abnormalities such as aneuploidy and wholechromosome loss of heterozygosity are commonly present in tumor cells. This suggests that chromosome instability and aneuploidy may play a critical role in tumor development and progression (Sen, 2000).

The integrity of the cell and of its genome and the correct accomplishment of cellular processes depend on the existence of control points in the cell cycle. These control mechanisms, called 'checkpoints', inhibit the transition to the next cell cycle phase if the events of the previous phase have not been correctly executed. A mitotic checkpoint has been identified that controls the metaphase to anaphase transition. A large number of studies have demonstrated that in vertebrates the kinetochore plays an active role in the mitotic checkpoint pathway and that microtubule accumulation at the kinetochore and/or tension that microtubules produce on the attached kinetochore can be the signal for mitotic checkpoint inactivation (Rieder and Salmon, 1998). Most of the mitotic checkpoint proteins localize on kinetochores during the early stages of mitosis (prophase and prometaphase) and disappear or become partially depleted from kinetochores before anaphase onset. These proteins can also be detected on unattached kinetochores of mono-oriented chromosomes at late prometaphase (Chen et al., 1996; Taylor and McKeon, 1997; Waters et al., 1998; Martinez-Esposito et al., 1999). The same behavior has been observed for the kinetochore phosphoepitopes recognized by the 3F3/2 antibody (Gorbsky and Ricketts, 1993; Campbell and Gorbsky, 1995). The mitotic checkpoint inhibits anaphase if spindle assembly is disrupted and if one or more kinetochores are not attached to spindle fibers. In the presence of these defects the mitotic checkpoint is activated, preventing anaphase until all kinetochores become attached (Rieder and Salmon, 1998). Hence, the activation of the mitotic checkpoint prevents the unbalanced distribution of chromosomes during mitosis and the production of aneuploid cells.

However, the fidelity of chromosome segregation is not absolute, and segregation defects, such as chromosomes left near the spindle equator after anaphase onset (lagging chromosomes) or sister chromatids migrated to the same pole (nondisjunction), have been observed in cultured mammalian 
cells (Ford et al., 1988; Cimini et al., 1999; Catalán et al., 2000). A large number of elegant studies in grasshopper meiosis, dating from more than two decades ago, resulted in a model in which, although initial kinetochore malorientations are very frequent, they are efficiently corrected during prometaphase by a process in which kinetochore microtubules can detach and reattach several times, until the correct orientation is achieved (Nicklas, 1971; Bajer and Molè-Bajer, 1972; Nicklas, 1997). This model proposes that meiotic or mitotic malsegregation may occur when kinetochore malorientations are not corrected before anaphase (Nicklas and Koch, 1969; Ault and Rieder, 1992).

Lagging chromosomes at anaphase represent a potential source of aneuploidy. After cytokinesis occurs, a lagging chromosome may give rise to a monosomic daughter cell and a trisomic one in $50 \%$ of cases. Several studies have shown that treatment with low doses of spindle poisons can induce chromosome loss during mitosis (Hsu and Satya-Prakash, 1985; Rizzoni et al., 1989; Marshall et al., 1996; Izzo et al., 1998; Cimini et al., 1999), and defects in chromosome segregation and lagging chromosomes at anaphase have been observed after inactivation of the mitotic checkpoint by antibody microinjection (Chan et al., 1999; Chan et al., 2000). Furthermore, cells subjected to prolonged exposure to high doses of spindle poisons are not permanently arrested but override the mitotic checkpoint and undergo a mitotic slippage (Andreassen and Margolis, 1994; Casenghi et al., 1999), suggesting that efficiency of the checkpoint may not be absolute. Both the meiotic studies and the more recent investigations on the mitotic checkpoint provided the ground for the recurrent idea in the literature that lagging chromosomes in mitosis may arise in cells that enter anaphase in the presence of mono-oriented chromosomes (Nicklas, 1971; Bajer and Molè-Bajer, 1972; Ford, 1985; Ault and Rieder, 1992; Chan et al., 2000).

We have recently shown for untreated PtK1 cells and PtK1 cells recovering from a mitotic block that lagging chromosomes in anaphase are single chromatids with only one kinetochore, as detected by CREST staining. Immunofluorescence and electron microscopy studies showed that the single kinetochore of a lagging chromosome is merotelically oriented (i.e. connected to microtubule bundles coming from both poles) (Cimini et al., 2001). In this work we extend our previous study by investigating whether human primary fibroblasts exhibit similar segregation errors and analyzing in live imaging experiments whether progression to anaphase of cells with mono-oriented chromosomes may contribute to the production of anaphase lagging chromosomes. In human cells, the use of chromosome-specific alphoid probes in fluorescence in situ hybridization experiments provides a sensitive tool to follow the distribution of single sisters from the same chromosome during mitosis and discriminate loss of a single sister from whole chromosome loss for specific chromosomes. Fluorescence in situ hybridization with chromosome 7 and 11 alphoid probes showed that single chromatids represent the great majority of loss events in ana-telophases for human primary fibroblasts released from a nocodazole-induced mitotic arrest. But loss of both sisters from the same chromosome is over-represented compared with the expected probability of a random occurrence of single events. We also analyzed the state of the mitotic checkpoint phosphoepitope recognized by the $3 F 3 / 2$ antibody on the kinetochores of lagging chromosomes in human anaphase cells. We found that they are devoid of the phosphoepitope, as expected if they attached microtubules and congressed to the metaphase plate prior to anaphase. Live cell imaging of mitotic progression in H2B-GFP-transfected human and PtK1 cells provided direct proof that lagging chromosomes observed in anaphase cells never derive from a checkpoint over-ride in the presence of mono-oriented chromosomes. Laggin chromosomes are seen left behind at the spindle equator during anaphase only, after metaphase chromosome congression, as predicted for lagging chromosomes having merotelic kinetochore orientation.

\section{Materials and Methods}

\section{Cell cultures}

Human lung diploid fibroblasts (MRC-5 cells) were obtained from the American Type Culture Collection (Rockville, MD) and maintained in Minimum Essential Medium (MEM) supplemented with $10 \%$ fetal calf serum, antibiotics and non-essential amino acids at $37^{\circ} \mathrm{C}, 5 \%$ $\mathrm{CO}_{2}$, in a humidified atmosphere. Potorous tridactylus kidney cells (PtK1) (American Type Culture Collection) were maintained in MEM supplemented with $10 \%$ fetal calf serum, antibiotics, non-essential amino acids, and $10 \mathrm{mM}$ Hepes at $37^{\circ} \mathrm{C}, 5 \% \mathrm{CO}_{2}$, in a humidified atmosphere.

\section{FISH analysis}

MRC-5 cells were treated with $35 \mathrm{ng} / \mathrm{ml}$ nocodazole (Sigma) for 16 hours to arrest cells in mitosis or with $0.125 \%$ DMSO, the nocodazole solvent, as control. At the end of the treatment, cells were washed three times in pre-warmed medium and then re-incubated in fresh medium at $37^{\circ} \mathrm{C}$ in order to release cells from the mitotic block. After 30 or 60 minutes, cultures were fixed in a 3:1 methanol:acetic acid mixture. FISH staining was performed using alphoid probes specific for chromosome 7 and 11 as previously described (Cimini et al., 1997). Preparations were examined using a Zeiss Axioplan microscope equipped with a $100 \times(1.3 \mathrm{NA})$ oil immersion objective and a CCD camera (Photometrics). DAPI, FITC and Rhodamine fluorescence were detected using the $0.1,10$ and 15 Zeiss filter sets, respectively. Grayscale images were acquired using IP Lab Spectrum software and processed with Adobe PhotoShop software. On each coverslip, all ana-telophase cells were visualized, and frequencies and types of lagging chromosomes were recorded. Frequencies of chromosome loss were analyzed by pooling data from cultures allowed to recover for 30 or 60 minutes after nocodazole treatment, since the cumulative frequency of ana-telophases observed at the two recovery times was found to correspond approximately to the frequency of mitotically arrested cells observed at the end of nocodazole treatment (data not shown). Data presented are the sum of two to four experiments.

\section{Immunostaining with 3F3/2 antibody}

MRC- 5 cells were treated with nocodazole for 16 hours, released for 30 or 60 minutes and then fixed and immunostained with the $3 \mathrm{~F} 3 / 2$ antibody. Cells were rapidly rinsed in PHEM buffer (60 $\mathrm{mM}$ Pipes, $25 \mathrm{mM}$ Hepes, pH 6.9, $10 \mathrm{mM}$ EGTA, $4 \mathrm{mM} \mathrm{MgSO}_{4}$ ) and then lysed for 5 minutes in $0.5 \%$ Triton-X in PHEM buffer in the presence of $100 \mathrm{nM}$ microcystin (Calbiochem). Cells were fixed for 20 minutes in $4 \%$ formaldehyde in PHEM, freshly prepared from paraformaldehyde, then rinsed in PBST (PBS with 0.05\% Tween-20) and subsequently blocked in 10\% boiled goat serum in PBS for 1 hour at room temperature. Coverslips were then incubated 2 hours at $37^{\circ} \mathrm{C}$ 
with the 3F3/2 antibody (a generous gift of G. J. Gorbsky, Health Sciences Center, University of Oklahoma, Oklahoma City, OK), rinsed in PBST and incubated with an FITC anti-mouse antibody (Vector Laboratories) diluted $1: 100$ in $5 \%$ boiled goat serum for 1 hour at $37^{\circ} \mathrm{C}$. Coverslips were rinsed again, counterstained with DAPI and mounted in an antifade solution (Vector Laboratories). Microscope observation and image acquisition were performed as described above.

\section{Analysis of mitotic progression and chromosome dynamics in live cells}

A plasmid carrying the full-length coding sequence for H2B histone subcloned into the pEGFP-N1 mammalian expression vector (a generous gift from Paulo Magalhaes, University of Padua, Italy) was used to express an H2B-GFP fusion protein under a CMV promoter in MRC-5 and PtK1 cells. MRC-5 cells, subcultured on $22 \mathrm{~mm}$ coverslips in $35 \mathrm{~mm}$ Petri dishes, were transiently transfected with 1 $\mu \mathrm{g}$ plasmid DNA using FuGENE 6 Transfection Reagent (Boheringer Mannheim). After 30 hours of incubation, cells were rinsed with PBS to remove the transfection mixture and incubated in $35 \mathrm{ng} / \mathrm{ml}$ nocodazole for 16 hours. Cells were then rinsed three times in medium and observed using fluorescence and phase-contrast microscopy after 30 minutes post-incubation in fresh medium. A Nikon Eclipse 300 inverted microscope equipped with a $37^{\circ} \mathrm{C}$ heated stage, $60 \times(0.7 \mathrm{NA})$ objective, and a Nikon B-2A filter block was used to follow live cell mitotic progression in MRC-5 cells. Late prometaphase or metaphase cells were localized and mitotic chromosome dynamics was observed. Time intervals from anaphase onset to cleavage furrow appearance and from cleavage furrow appearance to completion of cytokinesis were recorded. PtK1 cells were transfected as described above and culture medium was supplemented with $200 \mu \mathrm{g} / \mathrm{ml}$ Geneticin (Gibco BRL) 48 hours after transfection. Surviving cells at 1 week were maintained under selection using $60 \mu \mathrm{g} / \mathrm{ml}$ antibiotic. After 3 weeks the cell population was highly enriched $(>80 \%)$ in cells expressing the H2B-GFP fusion protein. PtK1 cultures enriched in H2B-GFP transfected cells were arrested in mitosis with $150 \mathrm{ng} / \mathrm{ml}$ nocodazole for 3 hours. Cells were subsequently washed, post-incubated in drugfree medium for 30 minutes and then observed by fluorescence and phase-contrast microscopy. A Nikon TE300 inverted microscope equipped with a $37^{\circ} \mathrm{C}$ heated stage, $100 \times(1.4 \mathrm{NA})$ Plan Apo phase 3 objective, a Chroma Hy-Q FITC filter set, and an Orca 1 CCD camera (Hamamatsu Photonics) was used to follow mitotic progression in living PtK1 cells by time-lapse microscopy. The microscope was controlled by MetaMorph imaging software (Universal Imaging Corp.).

\section{Results \\ Loss of a single sister is more frequent than loss of both sisters}

Chromosome loss events during mitosis can be visualized in anaphase cells as lagging chromosomes left near the spindle equator while all the other chromosomes correctly migrate to the spindle poles. In preliminary experiments human primary fibroblasts were arrested in prometaphase by incubation with the anti-microtubule drug nocodazole, then fixed after 30 or 60 minutes of growth in nocodazole-free medium and stained with DAPI. These experiments showed that in untreated human primary fibroblasts the frequency of anaphase lagging chromosomes for all the 23 chromosome pairs was $4.5 \%$ (per 1000), while in cells recovering from the nocodazole-induced mitotic block $40.9 \%$ anaphases showed one lagging chromosome. In addition, multiple lagging chromosomes were recorded in $33.4 \%$ anaphase cells after nocodazole treatment.
Therefore, the nocodazole treatment was used in order to enhance the incidence of lagging chromosomes at anaphase, due to its reversible microtubule disassembly activity. In situ hybridization using centromeric alphoid probes on anaphase cells was then used to investigate the nature of lagging chromosomes and the role of the mitotic checkpoint in chromosome loss during the release from a nocodazoleinduced mitotic block in human diploid cells. Hybridization with alphoid probes specific to chromosomes 7 and 11 allowed us to follow the distribution of these chromosomes in anaphase cells (Fig. 1A) and to discriminate single chromatid loss (Fig. 1B) from loss of both sister chromatids (Fig. 1C) for the two chromosomes under study. The analysis of centromeric hybridization signals showed that the majority of loss events consisted of single chromatids left behind at the cell equator (Fig. 1, graph). The frequency of single chromatid loss was $7.37 \%$ for chromosome 7 and $7.68 \%$ or for chromosome 11 , whereas lagging of two separated sisters from the same chromosome occurred at a frequency of $0.63 \%$ and $1.72 \%$ for chromosome 7 and 11, respectively. Loss of paired sister chromatids (Fig. 1D, graph) was instead a rare event, being observed in only 4 out of 130 analyzed chromosome loss events. Thus, kinetochore inactivation does not seem a relevant mechanism for chromosome malsegregation after antimicrotubule agents, since a higher frequency of paired sister chromatids would be expected if duplicated chromosomes did not congress to the metaphase plate due to lack of function of sister kinetochores. The higher frequencies of separated lagging sisters compared with paired lagging sisters also suggested that the absence of migration was not due to an inability of the chromosome to congress to the metaphase plate but, instead, was subsequent to anaphase onset and chromatid separation. Indeed, lagging chromosomes were always localized in the equatorial region of the cell, further indicating that they had congressed to the metaphase plate before anaphase onset.

Finally, a statistical analysis of the data showed that observed frequencies of loss of separated sisters from the same chromosome at anaphase were at least one order of magnitude higher than expected values for loss of single sisters from the same chromosome as independent events (chromosome 7 double loss expected value: $\left(7.37 \times 10^{-3}\right) \times\left(7.37 \times 10^{-3}\right)=$ $54.32 \times 10^{-6}=0.054 \%$; chromosome 7 experimental frequency: $0.63 \%$; chromosome 11 expected value: $\left(7.68 \times 10^{-3}\right) \times$ $\left(7.68 \times 10^{-3}\right)=58.98 \times 10^{-6}=0.059 \%$; $\quad$ chromosome 11 experimental frequency: $1.72 \%$ ), suggesting that the cellular mechanism responsible for chromosome loss during mitosis biases the random loss of sister chromatids.

\section{F3/2 antibody does not stain kinetochores of anaphase lagging chromosomes}

To investigate the state of an important mitotic checkpoint marker on anaphase lagging chromosomes in human primary fibroblasts, we immunostained MRC-5 cells with the 3F3/2 antibody, which recognizes a kinetochore phosphoepitope involved in the mitotic checkpoint signaling (Campbell and Gorbsky, 1995). Mono-oriented chromosomes possess this phosphoepitope on their kinetochores; it subsequently disappears as chromosomes bi-orient and properly align on the metaphase plate (Gorbsky and Ricketts, 1993; Nicklas et al., 

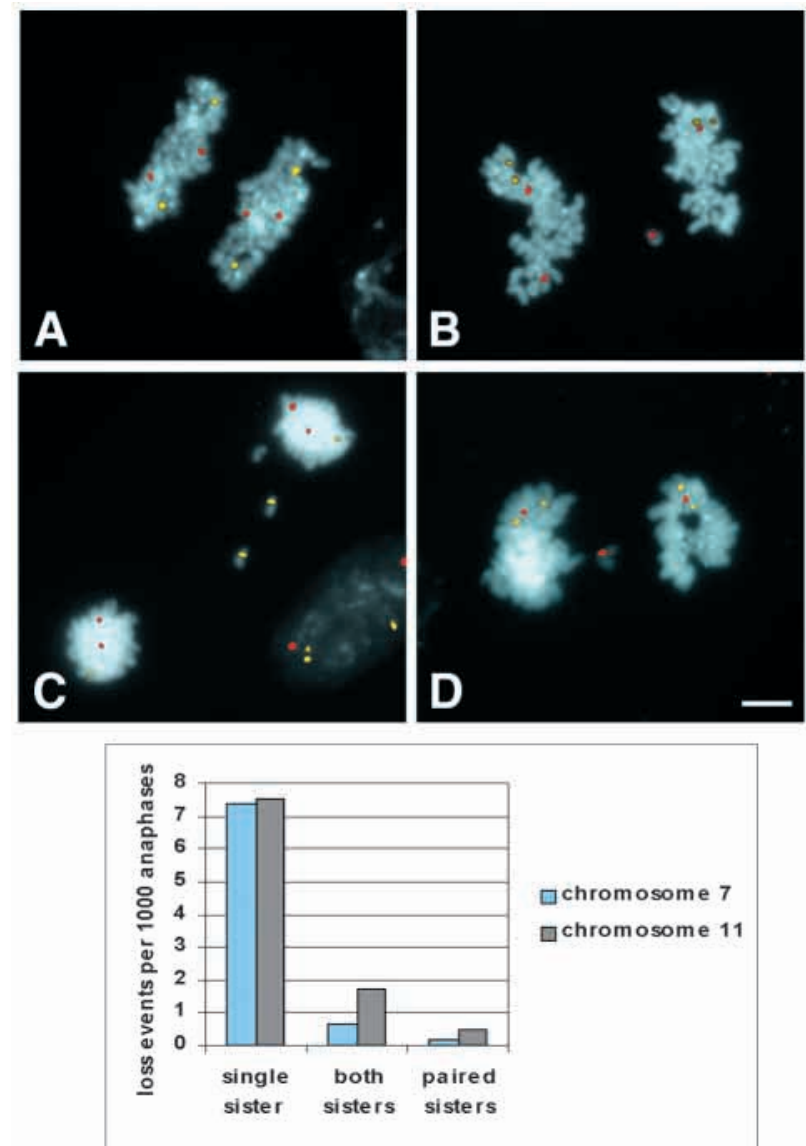

Fig. 1. FISH staining on anaphase human primary fibroblasts (MRC5 cells) recovering from a nocodazole-induced mitotic arrest. Alphoid-specific probes for chromosome 7 (FITC, yellow) and 11 (Rhodamine, red) were used. DNA was stained by DAPI (blue). (A) Normal distribution of chromosome 7 and 11 at spindle poles. (B) Loss of a single chromatid of chromosome 7. (C) Loss of both sister chromatids of chromosome 7 . When both sisters are lost in anaphase cells, their kinetochores are frequently separated and only in a few cases are the two chromatids still connected at the kinetochore level as shown in D for chromosome 11. The graph below shows quantitative results of the in situ hybridization analysis on 6378 anaphases, of which 94 displayed lagging chromosomes, for a total of 130 chromosome loss events for the two chromosomes under study. The data show that single lagging chromatids are the great majority of loss events. Bar, $5 \mu \mathrm{m}$.

1995). When tension is applied to a single unaligned chromosome by micromanipulation, 3F3/2 staining is lost and anaphase begins (Li and Nicklas, 1995; Li and Nicklas, 1997; Nicklas, 1997). Furthermore, when 3F3/2 antibody is injected into metaphase cells, anaphase onset is delayed (Campbell and Gorbsky, 1995).

MRC-5 cells were arrested in mitosis by nocodazole treatment and then fixed and immunostained after recovery in drug-free medium in order to characterize the $3 F 3 / 2$ behavior during mitosis in this human primary cell line and analyze 3F3/2 staining on lagging chromosomes at anaphase (Fig. 2). As already shown in other non-human and human cancer cell lines (Rieder and Salmon, 1998), unattached kinetochores from nocodazole-blocked MRC-5 cells were highly enriched in the
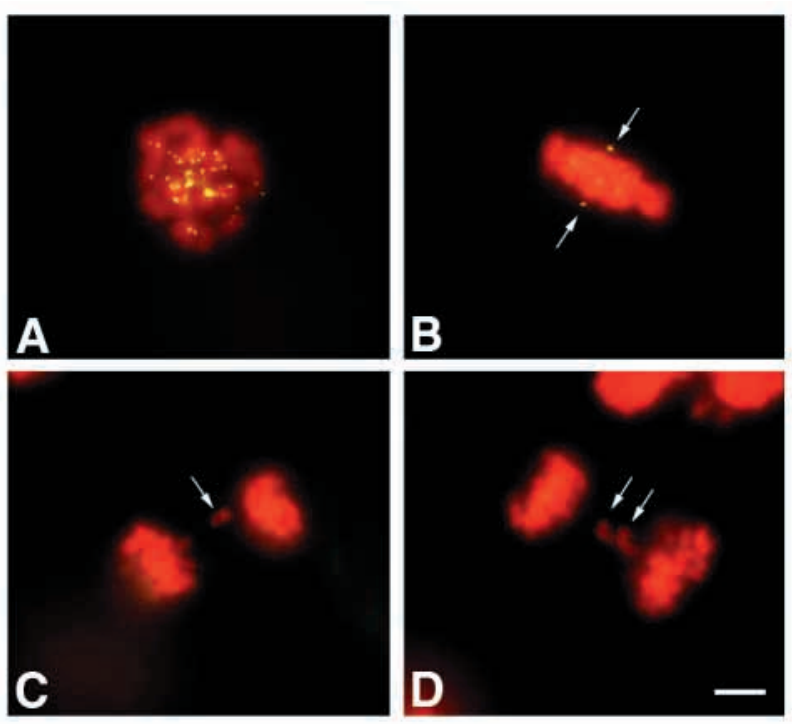

Fig. 2. 3F3/2 immunostaining on mitotic human primary fibroblasts (MRC-5 cells). (A) MRC-5 cell arrested in mitosis by nocodazole treatment. All the kinetochores show $3 \mathrm{~F} 3 / 2$ staining caused by the presence of the phosphoepitope recognized by the $3 F 3 / 2$ antibody on unattached kinetochores. (B) Metaphase cell after release from a nocodazole-induced mitotic block. The 3F3/2 antibody does not stain kinetochores, which are aligned at the metaphase plate and under tension. The 3F3/2 antibody does stain spindle poles (arrows). (C,D) Anaphase cells with lagging chromosomes (arrows) after release from the mitotic block. There is no $3 \mathrm{~F} 3 / 2$ staining on kinetochores of lagging chromosomes, nor on kinetochores of chromosomes correctly segregated to the spindle poles. DNA is pseudocolored in red; 3F3/2 antibody is shown in yellow. Bar, $5 \mu \mathrm{m}$.

3F3/2 phosphoepitope (Fig. 2A), whereas no staining was observed on aligned metaphase kinetochores (Fig. 2B). 3F3/2 immunodetection on human primary fibroblasts in anaphase showed that kinetochores of both segregated chromosomes and lagging chromosomes (346 cells analyzed) did not stain for the 3F3/2 antibody (Fig. 2C,D), as recently observed in PtK1 anaphase cells (Cimini et al., 2001). This suggests that kinetochores of lagging chromosomes observed in anaphase cells interacted with kinetochore microtubules at some point during prometaphase and/or metaphase, becoming dephosphorylated, as expected for kinetochores of bi-oriented chromosomes, which are under tension, and for merotelically oriented kinetochores. However, this analysis cannot exclude the possibility that during the release from the nocodazoleinduced mitotic arrest a fraction of cells over-rides the mitotic checkpoint in the presence of unattached or mono-oriented chromosomes and the phosphorylated epitope recognized by the $3 F 3 / 2$ antibody is dephosphorylated after anaphase onset.

\section{Lagging chromosomes appear during anaphase}

To test the possibility that cells showing lagging chromosomes at anaphase were cells progressing from metaphase to anaphase in the presence of mono-oriented chromosomes, we performed in vivo experiments to follow mitotic progression and chromosome dynamics in live cells after nocodazole exposure. In these experiments we considered chromosome alignment to the metaphase plate as representative of chromosome bi- 
Fig. 3. Chromosome dynamics during late mitotic stages in an MRC-5 cell expressing an H2B-GFP fusion protein. H2B-GFP-transfected MRC-5 cells were arrested in mitosis by nocodazole treatment and released in fresh medium for 30 minutes. From this time, late prometaphase or metaphase cells were localized and single cells were observed by fluorescence (A) and phase contrast (B) microscopy at regular intervals. Two lagging chromosomes are visible in anaphase/telophase (60 minutes, 70 minutes) after chromosome alignment at the metaphase plate had occurred (20 minutes). Numbers at the bottom right corner indicate the time in minutes. Bar, $5 \mu \mathrm{m}$.
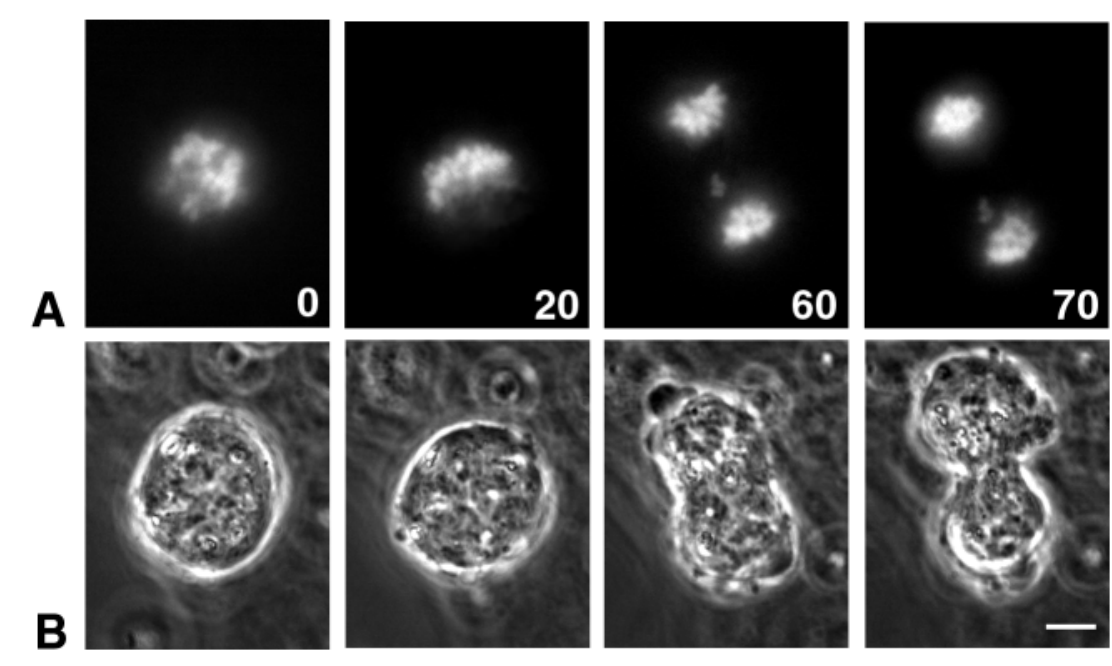
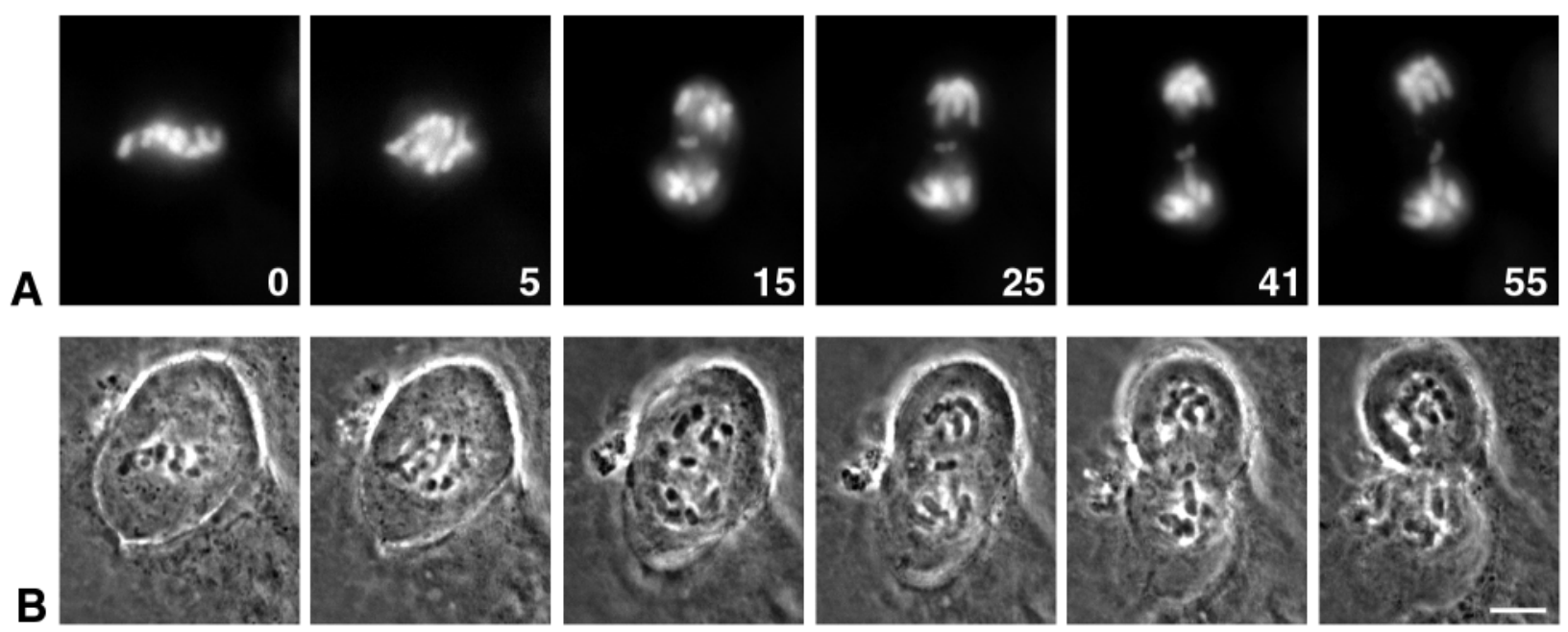

Fig. 4. Chromosome dynamics during late mitotic stages in an H2B-GFP-transfected PtK1 cell. H2B-GFP-transfected PtK1 cells were arrested in mitosis by nocodazole treatment and released in fresh medium for 30 minutes. From this time, late prometaphase or metaphase cells were localized and single cells were observed by fluorescence (A) and phase-contrast (B) microscopy at regular intervals. Numbers at the bottom right corner indicate the time in minutes. In the first frame ( 0 minutes), a PtK1 cell with all the chromosomes correctly aligned at the metaphase plate is shown; one lagging chromosome appears after anaphase onset (15 minutes) and persists as a lagging chromosome until late cytokinesis (55 minutes). Bar, $5 \mu \mathrm{m}$.

orientation, since live observations in other cell lines have shown that mono-oriented chromosomes are not aligned at the spindle equator but are closer to spindle poles; they then congress to the metaphase plate as they become bi-oriented (Rieder et al., 1994; Campbell and Gorbsky, 1995; Howell et al., 2000). The progression from metaphase to the completion of cytokinesis was followed in human primary fibroblasts recovering from a nocodazole-induced mitotic arrest, recording the time spent from anaphase onset to the appearance of the cleavage furrow (anaphase) and the time spent to complete cytokinesis after cleavage furrow appearance (telophase/ cytokinesis). To follow chromosome dynamics during mitosis, we transiently transfected human primary fibroblasts with a vector carrying an H2B-GFP fusion gene (Kanda et al., 1998). Transfected or untransfected human primary fibroblasts were nocodazole-arrested in mitosis and released for 30 minutes in fresh medium to allow spindle reassembly; late prometaphase or metaphase cells were then localized and observed until completion of cytokinesis by H2B-GFP fluorescence (Fig. 3A) and phase-contrast microscopy (Fig. 3B). The same type of experiment was performed on a PtK1 cell population highly enriched in transfected cells by non-clonal antibiotic selection after transfection (Fig. 4A (H2B-GFP fluorescence); Fig. 4B (phase contrast)). The use of a GFP-tagged histone construct in our work provided a powerful means to follow chromosome dynamics during mitosis. This was particularly effective in visualizing the mitotic chromosome behavior in the MRC-5 human fibroblast cell line, since the small size of human chromosomes, the three-dimensional shape of the mitotic cells, and the low contrast between chromosomes and cytoplasm makes the observation of chromosome dynamics by phasecontrast very ambiguous (Fig. 3B). The analysis of duration of mitosis in untransfected and H2B-GFP transfected MRC-5 cells showed that the expression of the H2B-GFP fusion protein does not affect the length of anaphase or telophase in cells recovering from the nocodazole-induced mitotic block 
Table 1. In vivo analysis of the late mitotic stages in human primary MRC-5 fibroblasts and PtK1 cells recovering from a nocodazole-induced mitotic arrest*

\begin{tabular}{|c|c|c|c|}
\hline & $\begin{array}{c}\text { Observed } \\
\text { mitotic cells }\end{array}$ & $\begin{array}{l}\text { Anaphase }{ }^{\dagger} \text { length } \\
\text { (mean minutes } \pm \text { s.d.) }\end{array}$ & $\begin{array}{l}\text { Telophase/cytokinesis length } \\
\text { (mean minutes } \pm \text { s.d.) }\end{array}$ \\
\hline \multicolumn{4}{|l|}{ MRC-5 cells } \\
\hline Untransfected & 25 & $13.04 \pm 5.22$ & $11.44 \pm 4.51$ \\
\hline H2B-GFP cells & 42 & $13.21 \pm 6.48$ & $10.26 \pm 4.23$ \\
\hline H2B-GFP cells with lagging chromosomes at anaphase/telophase & 5 & $13.00 \pm 6.89$ & $14.40 \pm 7.70$ \\
\hline \multicolumn{4}{|l|}{ PtK1 cells } \\
\hline H2B-GFP cells & 51 & $12.05 \pm 5.61$ & $11.17 \pm 8.30$ \\
\hline H2B-GFP cells with lagging chromosomes at anaphase/telophase & 12 & $14.00 \pm 11.13$ & $23.08 \pm 19.69^{\S}$ \\
\hline $\begin{array}{l}\text { *Nocodazole treatment: } 16 \text { hours with } 35 \mathrm{ng} / \mathrm{ml} \text { for MRC- } 5 \text { cells; } 3 \text { hour } \\
\text { †From anaphase onset to cleavage furrow appearance. } \\
\text { } \text { From cleavage furrow appearance to completion of cytokinesis. } \\
\$_{P<0.01 \text { comparing telophase/cytokinesis length in PtK1 cells with and }}\end{array}$ & th $150 \mathrm{ng} / \mathrm{ml} \mathrm{fc}$ & omes by a Student & \\
\hline
\end{tabular}

Fig. 5. Analysis of lagging chromosome behavior during cell cleavage by time-lapse microscopy in a PtK1 cell released from a nocodazoleinduced mitotic arrest. Numbers at the bottom right corner indicate the time in minutes from nocodazole washout. Two lagging chromosomes can be seen in the equatorial region of the dividing cell from cleavage furrow formation (60 minutes)
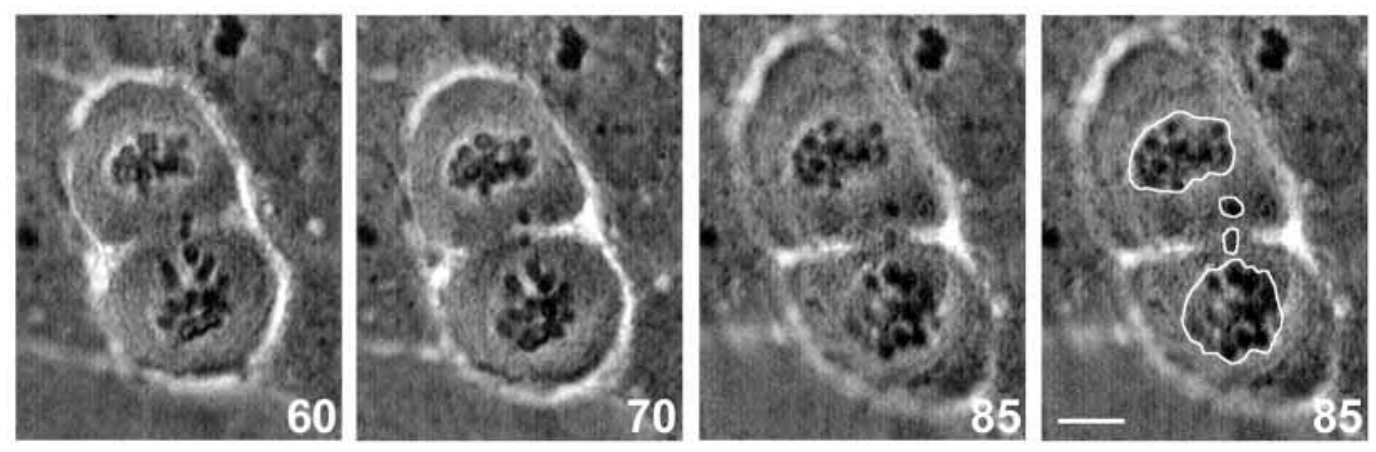
micronuclei deriving from the two lagging chromosomes is highlighted by a white line for clarity. Bar, $5 \mu \mathrm{m}$.

(Table 1). The analysis of mitotic progression in H2B-GFP expressing MRC-5 and PtK1 cells provided important clues for understanding the dynamics of events leading to lagging chromosomes at anaphase and the fate of these malsegregated chromosomes. First, none of the cells that showed one or a few unaligned chromosomes entered anaphase in MRC-5 cells (5 out of 5 cells), or in PtK1 cells (7 out of 7 cells) within the period of live observation (2-3 hours). Second, all lagging chromosomes that could be observed at anaphase appeared in cells in which all the chromosomes correctly aligned at the metaphase plate before anaphase onset (MRC-5 cells: see 60 and 20 minutes in Fig. 3; PtK1 cells: see 15 and 0 minutes in Fig. 4). Third, none of the lagging chromosomes we observed was incorporated in the daughter nuclei during nuclear envelope reformation but instead formed micronuclei (Fig. 5). This suggests that the potential aneuploidy caused by lagging chromosomes cannot be rescued by successful later migration or by passive incorporation of lagging chromosomes into the main nucleus at the end of mitosis. As a whole, the live observation of mitotic progression of cells with lagging chromosomes demonstrated that in both cell lines the mitotic checkpoint is fully proficient in blocking the metaphase to anaphase transition in cells with mono-oriented chromosomes. However, it does not prevent the loss of genetic information represented by merotelically oriented lagging chromosomes.

The comparison of anaphase and telophase length did not show statistically significant differences between MRC-5 cells with and without lagging chromosomes at anaphase, although a tendency towards longer time in telophase/cytokinesis for cells with lagging chromosomes was observed (Table 1). In PtK1 cells the presence of lagging chromosomes did not affect anaphase length but significantly delayed the completion of cytokinesis (Table 1), suggesting that cytokinesis rather than chromosome migration is affected by the presence of lagging chromosomes in this cell line. It should be noted that the numbers reported as telophase/cytokinesis times in our live observations refer to the minutes elapsed between cleavage furrow formation and the completion of cell cleavage and therefore the measurements reflect more completion of cytokinesis than chromosome migration, which is usually completed before the cleavage furrow forms. In a number of cases we observed chromosomes migrating behind the others that successively reached the segregated chromosomes at one pole, but in these cells anaphase and telophase/cytokinesis length was unaffected (data not shown).

\section{Discussion}

Both in yeast and vertebrates the mitotic spindle checkpoint has been shown to efficiently prevent the metaphase to anaphase transition in the presence of an impaired mitotic spindle or in the presence of unattached kinetochores (Burke, 2000). Nevertheless, lagging chromosomes at anaphase can be observed in cultured cells (Ford et al., 1988; Izzo et al., 1998; Cimini et al., 1999; Catalán et al., 2000). We recently showed that lagging chromosomes observed in fixed preparations of PtK1 anaphase cells are single chromatids with merotelically oriented kinetochores (Cimini et al., 2001). The present study 

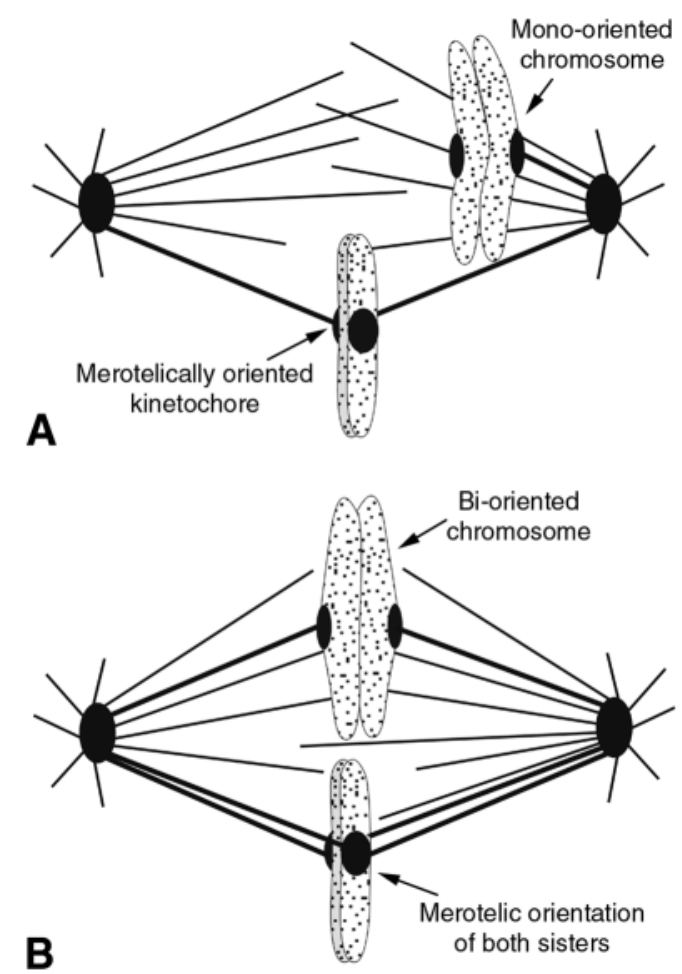

Fig. 6. Proposed events leading to the preferential loss of both sister chromatids at anaphase. Acquisition of a merotelic orientation of one sister kinetochore twists the position of the whole chromosome (A), promoting the merotelic orientation of the other sister kinetochore (B). In mono-oriented chromosomes, sister kinetochores face opposite poles (A), favoring chromosome bi-orientation (B).

extends this previous one by showing that, in human primary cells, anaphase lagging chromosomes are also single chromatids devoid of $3 \mathrm{~F} 3 / 2$ staining, providing further evidence that merotelic orientation is the kinetochore defect producing lagging chromosomes and that this kinetochore malorientation is not detected by the mitotic spindle checkpoint. The use of centromeric FISH staining on anaphase human cells enabled us to follow the distribution of sister chromatids during mitosis and to observe an overrepresentation of separated sisters from the same chromosome among double lagging (Fig. 1). This is an interesting finding, since it suggests that bipolar attachment of a single kinetochore (merotelic orientation) may also alter the position of the other sister kinetochore with respect to microtubules and promote the merotelic orientation of the other sister kinetochore (Fig. 6). This is readily understood if one considers that acquisition of a merotelic configuration of a single kinetochore twists the position of the whole chromosome within the spindle architecture (Fig. 6A) in such a way that the other sister no longer faces the opposite pole, but is prone to interact with microtubules from both poles (Fig. 6B).

In the present study the in vivo observation of mitotic progression during recovery from nocodazole demonstrates that lagging chromosomes in anaphase are observed in cells where all the chromosomes correctly aligned at the metaphase plate. This was found in both PtK1 and human cells, providing a general significance to the conclusion that chromosome loss in cultured tissue cells is not due to an over-riding of the mitotic checkpoint in the presence of mono-oriented kinetochores. Our live cell imaging shows that the mitotic checkpoint efficiently inhibits anaphase onset in cells with unattached kinetochores both in human and PtK1 cell lines, thereby preventing the possible aneuploid burden caused by mono-oriented chromosomes. However, this control mechanism does not detect kinetochore merotelic orientation, since this unusual interaction between kinetochores and kinetochore microtubules inactivates the checkpoint, as shown in this and previous work (Cimini et al., 2001). Earlier live cell experiments had already shown merotelic orientation of chromosome fragments possessing one kinetochore lagging behind at anaphase (Khodjakov et al., 1997) or of chromosomes stranded at the spindle equator in anaphase cells with multipolar spindles (Sluder et al., 1997).

A crucial question for understanding the origin of mitotic instability is whether the presence of a lagging chromosome in anaphase may activate a kind of control mechanism, allowing time for correction of this segregation error. In fission yeast, slowing of spindle elongation in live cells with lagging chromosomes was recently observed by time-lapse microscopy (Pidoux et al., 2000). However, no clear indication for a possible 'anaphase checkpoint' was provided in that paper, which also showed, consistently with our 3F3/2 data, that the mitotic checkpoint protein Bub1 was not present on lagging chromosomes at anaphase (Pidoux et al., 2000). Our data suggest that an active mechanism that delays anaphase/ telophase in cells with lagging chromosomes is not likely, since no significant lengthening of the time between anaphase onset and cleavage furrow formation is observed in human fibroblasts or PtK1 cells that show lagging chromosomes at anaphase. By contrast, a significant lengthening of the cleavage process was observed in PtK1 cells. This might be related to the large size of chromosomes in PtK1 cells, which renders this cell line a paradigmatic experimental model for studying mitosis and chromosome segregation. In PtK1 cells the presence of a lagging chromosome may influence the duration of mitosis by exerting a mechanical impediment to cleavage furrow ingression and cytokinesis completion, whereas in human cells this may depend very much on the size of the lost chromosome. Thus, we propose cell cleavage delay rather than activation of an 'anaphase/telophase checkpoint', as the cause of telophase/cytokinesis lengthening in PtK1 cells with lagging chromosomes. This is also consistent with the observation that cytokinesis completion is longer in PtK1 cells with more than one lagging chromosome compared with cells with a single lagging chromosome (single lagging: 20.29 \pm 16.22 minutes; multiple lagging: $27.8 \pm 25.9$ minutes).

It has been recognized for many years that micronuclei result as the consequence of the exclusion from daughter nuclei of acentric fragments or whole chromosomes lagging at anaphase (Evans, 1988; Ford et al., 1988; Heddle et al., 1991). The quantitative relationship between frequencies of acentric fragments and micronuclei is not precisely known, although it is suggested that between 30 and $70 \%$ of the acentric fragments seen in anaphase become micronuclei (Evans, 1988; Heddle et al., 1991). Frequencies of the same order of magnitude between whole chromosome lagging at anaphase and micronuclei have been observed in human lymphocytes, suggesting that a large part of lagging chromosomes give rise to centromere positive micronuclei (Ford et al., 1988; 
Gustavino et al., 1994). Our in vivo analysis is consistent with the quantitative relationship between lagging chromosomes and micronuclei inferred from analyses on fixed preparations. This is a relevant aspect since it means that the presence of a lagging chromosome at anaphase may lead, in half of cases, to the formation of a monosomic daughter cell and a trisomic one possessing a disomic nucleus and an extra chromosome in a micronucleus. It has also been shown that some micronuclei can perform DNA synthesis and mitotic condensation synchronously with the main nucleus, further producing chromosome loss and gain at successive mitotic cycles (Rizzoni et al., 1989; Gustavino et al., 1994; Minissi et al., 1999). This may explain the generation of chromosome instability after chromosome loss. Accordingly, the presence of micronuclei in interphase appears to be a common feature of tumor cells (Saunders et al., 2000; Sen, 2000).

In conclusion, our results demonstrate that the mitotic checkpoint efficiently prevents the possible aneuploid burden caused by mono-oriented chromosomes in human primary fibroblast cells and that merotelic kinetochore orientation is a major limitation for accurate chromosome segregation and a potentially important mechanism of aneuploidy in human cells. The identification of cellular mechanisms involved in chromosome malsegregation is of great interest because of the potential role that aneuploidy has been suggested to have in tumor development and progression (Li et al., 1997; Duesberg et al., 1998; Cahill et al., 1998; Lengauer et al., 1998; Lee et al., 1999). It represents an extraordinarily important clue to understand the genesis of chromosome instability in tumor cells and a starting point for the development of new therapeutic strategies.

We thank G. J. Gorbsky for the gift of the 3F3/2 antibody and P. Magalhaes for the H2B-GFP vector. D.C. is a recipient of a fellowship from the Italian Federation for Cancer Research (FIRC). This work was also supported by NIH GM24364 to E.D.S.

\section{References}

Andreassen, P. R. and Margolis, R. L. (1994). Microtubule dependency of p34cdc2 inactivation and mitotic exit in mammalian cells. J. Cell Biol. 127, 789-802.

Ault, J. G. and Rieder, C. L. (1992). Chromosome mal-orientation and reorientation during mitosis. Cell Motil. Cytoskel. 22, 155-159.

Bajer, A. S. and Molè-Bajer, J. (1972). Hypotheses of chromosome movements and spindle structures. Int. Rev. Cyt. Suppl. 3, 177-215.

Burke, D. J. (2000). Complexity in the spindle checkpoint. Curr. Opin. Genet. Dev. 10, 26-31.

Cahill, D. P., Lengauer, C., Yu, J., Riggens, G. J., Willson, J. K. V., Markowitz, S. D., Kinzler, K. W. and Vogelstein, B. (1998). Mutations of mitotic checkpoint genes in human cancers. Nature 392, 300-303.

Campbell, M. S. and Gorbsky, G. J. (1995). Microinjection of mitotic cells with the 3F3/2 anti-phosphoepitope antibody delays the onset of anaphase. J. Cell Biol. 129, 1195-1204.

Casenghi, M., Mangiacasale, R., Tuynder, M., Caillet-Fauquet, P., Elhajouji, A., Lavia, P., Mousset, S., Kirsch-Volders, M. and Cundari, E. (1999). p53-independent apoptosis and p53-dependent block of DNA rereplication following mitotic spindle inhibition in human cells. Exp. Cell Res. 250, 339-350.

Catalán, J., Falck, G. C.-M. and Norppa, H. (2000). The X chromosome frequently lags behind in female lymphocyte anaphase. Am. J. Hum. Genet. 66, 687-691.

Chan, G. K. T., Jablonski, S. A., Sudakin, V., Hittle, J. C. and Yen, T. J. (1999). Human BUBR1 is a mitotic checkpoint kinase that monitors CENPE functions at kinetochores and binds the cyclosome/APC. J. Cell Biol. 146, 941-954.
Chan, G. K. T., Jablonski, S. A., Starr, D. A., Goldberg, M. L. and Yen, T. J. (2000). Human Zw10 and ROD are mitotic checkpoint proteins that bind to kinetochores. Nat. Cell Biol. 2, 944-947.

Chen, R. H., Waters, J. C., Salmon, E. D. and Murray, A. W. (1996). Association of spindle checkpoint component XMAD2 with unattached kinetochores. Science 274, 242-246.

Cimini, D., Antoccia, A., Tanzarella, C. and Degrassi, F. (1997). Topoisomerase II inhibition in mitosis produces numerical and structural chromosomal aberrations in human fibroblasts. Cytogenet. Cell Genet. 76, 61-67.

Cimini, D., Tanzarella, C. and Degrassi, F. (1999). Differences in malsegregation rates obtained by scoring ana-telophase or binucleate cells. Mutagenesis 14, 563-568.

Cimini, D., Howell, B., Maddox, P., Khodjakov, A., Degrassi, F. and Salmon, E. D. (2001). Merotelic kinetochore orientation is a major mechanism of aneuploidy in mitotic mammalian tissue cells. J. Cell Biol. 153, 517-527.

Duesberg, P., Rausch, C., Rasnik, D. and Helmann, R. (1998). Genetic instability of cancer cells is proportional to their degree of aneuploidy. Proc. Natl. Acad. Sci. USA 95, 13692-13697.

Evans, H. J. (1988). Mutation cytogenetics: past, present and future. Mutat. Res. 204, 355-363.

Ford, J. H. (1985). A model for the mechanism of aneuploidy involving chromosome displacement. In Aneuploidy: Etiology and Mechanisms. (ed. V. L. Dellarco, P. E. Voytek and A. Hollaender), pp. 291-295. Plenum Press, New York.

Ford, J. H., Schultz, C. J. and Correll, A. T. (1988). Chromosome elimination in micronuclei: a common cause of hypoploidy. Am. J. Hum. Genet. 43, 733-740.

Gorbsky, G. J. and Ricketts, W. A. (1993). Differential expression of a phosphoepitope at the kinetochores of moving chromosomes. J. Cell Biol. 122, 1311-1321.

Gustavino, B., Degrassi, F., Filipponi, R., Modesti, D., Tanzarella, C. and Rizzoni, M. (1994). Mitotic indirect non-disjunction in phytohemagglutinin stimulated human lymphocytes. Mutagenesis 9, 17-21.

Heddle, J. A., Cimino, M. C., Hayashi, M., Romagna, F., Shelby, M. D., Tucker, J. D., Vanparys, P. and MacGregor, J. T. (1991). Micronuclei as an index of cytogenetic damage: past, present, and future. Environ. Mol. Mutagen. 18, 277-291.

Howell, B. J., Hoffman, D. B., Fang, G., Murray, A. W. and Salmon, E. D. (2000). Visualization of Mad2 dynamics at kinetochores, along spindle fibers, and at spindle poles in living cells. J. Cell Biol. 150, 1233-1250.

Hsu, T. C. and Satya-Prakash, K. L. (1985). Aneuploidy induction by mitotic arrestants in animal cell systems: possible mechanisms. In Aneuploidy Etiology and Mechanisms (ed. V. L. Dellarco, P. E. Voytek and A. Hollaender), pp. 279-289. Plenum Press, New York.

Izzo, M., Antoccia, A., Degrassi, F. and Tanzarella, C. (1998). Immunofluorescence analysis of diazepam-induced mitotic apparatus anomalies and chromosome loss in Chinese hamster cells. Mutagenesis 13, 445-451.

Kanda, T., Sullivan, K. F. and Wahl, G. M. (1998). Histone-GFP fusion protein enables sensitive analysis of chromosome dynamics in living mammalian cells. Curr. Biol. 8, 377-385.

Khodjakov, A., Cole, R. W., McEwen, B. F., Buttle, K. F. and Rieder, C. L. (1997). Chromosome fragments possessing only one kinetochore can congress to the spindle equator. J. Cell Biol. 136, 229-240.

Lee, H., Trainer, A. H., Friedman, L. S., Thistlethwaite, F. C., Evans, M. J., Ponder, B. A. and Venkitaraman, A. R. (1999). Mitotic checkpoint inactivation fosters transformation in cells lacking the breast cancer susceptibility gene, Brca2. Mol. Cell 4, 1-10.

Lengauer, C., Kinzler, K. W. and Vogelstein, B. (1998). Genetic instabilities in human cancers. Nature 396, 643-649.

Li, R., Yerganian, G., Duesberg, P., Kraemer, A., Willer, A., Rausch, C. and Hehlmann, R. (1997). Aneuploidy correlated $100 \%$ with chemical transformation of Chinese hamster cells. Proc. Natl. Acad. Sci. USA 94, 14506-14511.

Li, X. and Nicklas, R. B. (1995). Mitotic forces control a cell-cycle checkpoint. Nature 373, 630-632.

Li, X. and Nicklas, R. B. (1997). Tension-sensitive kinetochore phosphorylation and the chromosome distribution checkpoint in praying mantid spermatocytes. J. Cell Sci. 110, 537-545.

Marshall, R. R., Murphy, M., Kirkland, D. J. and Bentley, K. S. (1996). Fluorescence in situ hybridisation with chromosome-specific centromeric probes: a sensitive method to detect aneuploidy. Mutat. Res. 372, 233-245. 
Martinez-Esposito, M. J., Kaplan, K. B., Copeland, J. and Sorger, P. K. (1999). Retention of the Bub3 checkpoint protein on lagging chromosomes. Proc. Natl. Acad. Sci. USA 96, 8493-8498.

Minissi, S., Degrassi, F., Tanzarella, C. and Gustavino, B. (1999). Direct and indirect non-disjunction in the origin of trisomy in cultured human lymphocytes. Mutagenesis 14, 557-562.

Nicklas, R. B. (1971). Mitosis. Adv. Cell Biol. 2, 225-297.

Nicklas, R. B. (1997). How cells get the right chromosomes. Science 275, 632637.

Nicklas, R. B. and Koch, C. A. (1969). Chromosome micromanipulation. 3. Spindle fiber tension and the reorientation of mal-oriented chromosomes. $J$. Cell Biol. 43, 40-50.

Nicklas, R. B., Ward, S. G. and Gorbsky, G. J. (1995). Kinetochore chemistry is sensitive to tension and may link mitotic forces to a cell cycle checkpoint. J. Cell Biol. 130, 929-939.

Pidoux, A. L., Uzawa, S., Perry, P. E., Cande, W. Z. and Allshire, R. C. (2000). Live analysis of lagging chromosomes during anaphase and their effect on spindle elongation rate in fission yeast. J. Cell Sci. 113, 4177-4191.

Rieder, C. L. and Salmon, E. D. (1998). The vertebrate cell kinetochore and its roles during mitosis. Trends Cell Biol. 8, 310-318.
Rieder, C. L., Schultz, A., Cole, R. and Sluder, G. (1994). Anaphase onset in vertebrate somatic cells is controlled by a checkpoint that monitors sister kinetochore attachment to the spindle. J. Cell Biol. 127, 1301-1310.

Rizzoni, M., Tanzarella, C., Gustavino, B., Degrassi, F., Guarino, A. and Vitagliano, E. (1989). Indirect mitotic non disjunction in Vicia faba and Chinese hamster cells. Chromosoma 97, 339-346.

Saunders, W. S., Shuster, M., Huang, X., Gharaibeh, B., Enyenihi, A. K., Petersen, I. and Gollin, S. M. (2000). Chromosomal instability and cytoskeletal defects in oral cancer cells. Proc. Natl. Acad. Sci. USA 97, 303308 .

Sen, S. (2000). Aneuploidy and cancer. Curr. Opin. Oncol. 12, 82-88.

Sluder, G., Thompson, E. A., Miller, F. J., Hayes, J. and Rieder, C. L. (1997) The checkpoint control for anaphase onset does not monitor excess numbers of spindle poles or bipolar spindle symmetry. J. Cell Sci. 110, 421-429.

Taylor, S. S. and McKeon, F. (1997). Kinetochore localization of murine Bub1 is required for normal mitotic timing and checkpoint response to spindle damage. Cell 89, 727-735.

Waters, J. C., Chen, R.-H., Murray, A. W. and Salmon, E. D. (1998). Localization of Mad2 to kinetochores depends on microtubule attachment, not tension. J. Cell Biol. 141, 1181-1191. 